\title{
Properties of satellite galaxies in nearby groups
}

\author{
Jaan Vennik \\ Tartu Observatory, 61602 Tõravere, Tartumaa, Estonia \\ email: vennik@to.ee
}

\begin{abstract}
We studied the variation of stellar mass and various star-formation characteristics of satellite galaxies in a volume limited sample of nearby groups as a function of their group-centric distance and of their relative line-of-sight velocity in the group rest frame. We found clear radial dependencies, e.g. massive, red and passive satellites being distributed predominantly near the center of composite group. We also found some evidence of velocity modulation of star-forming properties of satellite galaxies near the group virial radius. We conclude that using kinematical data, it should be feasible to separate dynamical classes of bound, in-falling and 'backsplash' satellite galaxies.
\end{abstract}

Keywords. galaxies: clusters: general, galaxies: statistics, galaxies: evolution

\section{Introduction}

Satellite galaxies are proper test bodies when studying the physical mechanisms that drive the galaxy evolution in groups and clusters. The star-forming in-falling galaxies are expected to have distinctive properties compared to the passively evolving partly virialised population near the cluster/group center. An intermediate population of 'backsplash' galaxies may have lost a significant fraction of their mass and velocity by crossing the central region and hence they may contribute to the radial velocity segregation in galaxy properties on the outskirts of galaxy groups and clusters. Earlier studies have found statistically significant evidence of velocity segregation of galaxy properties in clusters (e.g. Mahajan et al. 2011). According to the group pre-processing scenario of cluster galaxies, similar processes can take place also in groups of galaxies.

\section{Data and analysis}

Our target groups are extracted from the parent catalogue of groups and clusters of galaxies, based on the spectroscopic sample of the SDSS DR10 (Tempel et al. 2014). We selected a sample of 2112 medium richness $\left(5 \leqslant n_{\text {gal }}<50\right)$, relatively nearby $(0.013<$ $z<0.05)$ groups, for which are available re-processed photometric data (Blanton et al. 2011) in the NASA-Sloan Atlas (http://nsatlas.org). We use also the galaxy stellar masses $\left(M^{*}\right)$, specific star-formation rates (sSFR) and various spectral characteristics $(\mathrm{EW}(\mathrm{H} \alpha)$, $\mathrm{EW}(\mathrm{H} \delta)$, Dn4000 break), obtained from the SDSS CAS.

Our aim is to address the radial trends of various physical and star-formation characteristics of satellite galaxies in groups, and the possible velocity modulations of these trends, conditioned by dynamical evolution in the group core and by occurence of infalling and 'backsplash' galaxies in radial range of about 1-3 virial radii. For this purpose we stacked together the complete sample of 16284 satellite (rank $>1$ ) galaxies, with limiting absolute magnitudes of $M_{r}<-18$, using the group-centric distances, normalized by the virial radius $\left(R_{v i r}\right)$ and relative line-of-sight $(l o s)$ velocities scaled by the 

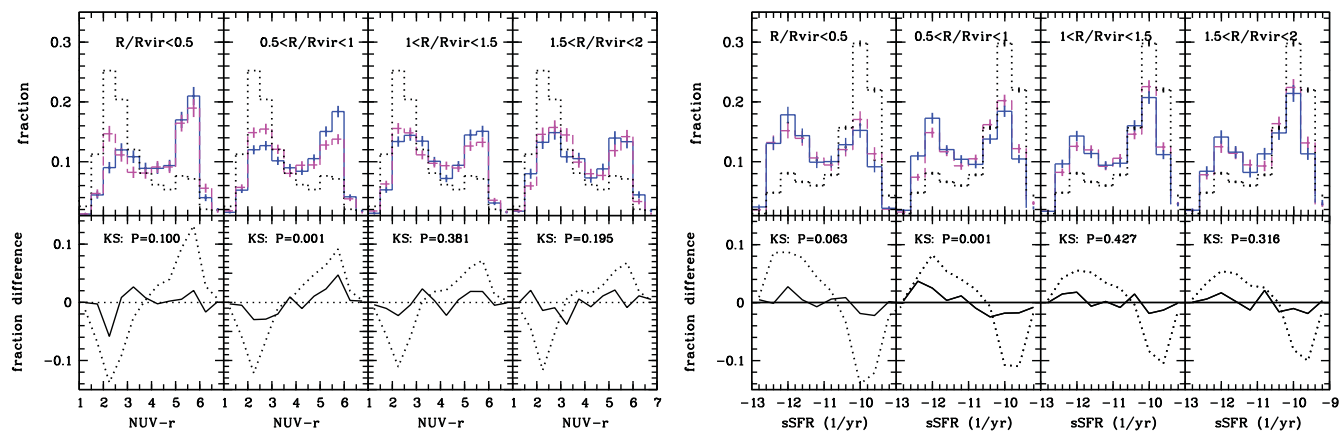

Figure 1. The fraction distributions of the (NUV-r) colours (left panel) and the specific SFRs (right panel) of low- and high-velocity satellite galaxies in four scaled radial bins are compared to one another and to the properties of field galaxies. Upper section: Solid (blue) histogram represents satellites with low velocities, dashed (mageneta) histogram is for high velocity satellites; dotted (black) histogram denotes the field galaxies. Error bars are Poisson. Lower section: shows the fraction difference between two velocity bins $(\operatorname{frac}(<0.75)-\operatorname{frac}(>0.75)$ - solid line $)$ and the difference between all satellites and field galaxies ( frac(sat.) - frac(field) - dotted line). The Kolmogorov-Smirnov (KS) test probabilities (P) for the null hypothesis is indicated for the distribution of satellites in two velocity bins. Fraction of red/passive and blue/star-forming satellite galaxies is changing with group-centric radius. There is an excess of blue high velocity satellites near the center and an excess of red low velocity satellites near the group virial radius. The fraction distributions of properties of field and satellite galaxies are different, however the difference decreases for satellites, which are located at the group outskirts.

velocity dispersion $\left(\sigma_{v}\right)$ of each group. For comparison purposes we selected a similar sample of field ( rank $=0$ ) galaxies from the same parent catalogue. We analyse the fraction distribution for various properties of low-velocity $\left(|v-<v>| / \sigma_{v}<0.75\right)$ and high-velocity $\left(\mid v-\langle v>| / \sigma_{v}>0.75\right)$ satellite galaxies in four scaled radial bins $\left(R / R_{\text {vir }}=0-0.5,0.5-1.0,1.0-1.5,1.5-2\right)$.

\section{Results}

We found statistically significant radial dependencies of satellite properties, e.g. massive, red and passive satellites being distributed predominantly near the center of the composite group. Our study also highlights some velocity modulation of these radial trends, e.g. (i) a mild excess of massive satellites in the group core, and (ii) an excess of red and passive satellites near the group virial radius, amongst the low velocity satellite galaxies (Fig. 1). We conclude that the galaxy properties in and around groups are shaped not only by their stellar mass and environment but also by absolute los velocity. Using kinematical data, it should be statistically feasible to separate dynamical classes of bound, in-falling and 'backsplash' satellite galaxies also in poor groups of galaxies.

\section{References}

Blanton, M. R., Kazin, E., Muna, D., Weaver, B. A., \& Price-Whelan, A. 2011,

Mahajan, S., Mamon, G. A., \& Raychaudhury, S. 2011, MNRAS, 416, 2882

Tempel, E., Tamm, A., Gramann, M., et al. 2014, A\&A, 566, A1 